Research letter

\section{Follow-up of the Finnish \\ Asthma Programme 2000-2010: reduction of hospital burden needs risk group rethinking}

The Finnish Asthma Programme 1994-2004 focused on early intervention and disease control, thereby resulting in a significant reduction of asthma morbidity. During the follow-up period from 2000 to 2010, the number of hospital days continued to fall by $54 \%$. Patients $\geq 65$ years, especially women, accounted for $39 \%$ of the hospital days, and they need attention if the hospital burden is to be reduced further.

In Finland, which has a total population of 5.4 million, 191000 asthma patients in 2000 and 233000 (4\% of the population) in 2010 (a 22\% increase) were entitled to a special reimbursement of drug costs, according to the Social Insurance Institution (figure 1A). In adults, after hypertension, asthma was the second most common chronic disorder in the reimbursement register, indicating the need for regular medication. In children $\leq 15$ years, asthma was the most frequently compensated chronic disorder. In Helsinki, the prevalence of self-reported physician-diagnosed asthma in adults has increased from $6.8 \%$ in 1996 to $9.4 \%$ in $2007 .^{1}$

The improved care provided by the Finnish Asthma Programme 1994-2004 resulted in a reduction of the disease burden; for example, the yearly total costs for asthma were reduced by one-third. ${ }^{2}$ Similarly, the Finnish Allergy Programme 2008-2018 aimed to reduce emergency visits due to asthma by $40 \% .^{3}$ In this study, we report the temporal trends of hospital use for asthma in the 11-year period from 2000 to 2010.

The patient numbers and hospital days were derived from the Finnish Hospital Discharge Register by the National Institute for Health and Welfare (ICD-10 J45-J46). The data for chronic obstructive pulmonary disease (COPD; ICD-10 J44) were compared for reference. The 2010 data for asthma were analysed by age groups.

In 2000, 5894 patients diagnosed with asthma used 32000 hospital days compared with 2938 patients using 15000 hospital days in 2010 (an absolute decrease of $54 \% ; 65 \%$ taking into account the number of patients with asthma in the reimbursement register (figure 1A)). The average stay in hospital was reduced from 4.3 days to 4.2 days. For patients diagnosed with COPD, the hospital days were reduced from 44000 to 23000 (an absolute decrease of $46 \%$; figure 1A). In 2010, 39\% of the hospital days were due to asthma and $61 \%$ were due to COPD. During the study period, any diagnostic transfer between asthma and COPD is unlikely as the hospital days decreased at a similar rate and diagnostic practices did not change.

In 2010, 39\% of the hospital days for asthma were used by patients $\geq 65$ years old (figure 1B), $15 \%$ by patients $\leq 15$ years old and $12 \%$ by children $\leq 5$ years old (figure 1C). However, in the case of older patients, the number of hospital days decreased by $60 \%$ during the study period. Older patients with asthma, especially women, needed attention, since the majority of the hospitalisations involved women $\geq 65$ years old (figure 1D). Co-morbidities of asthma, such as COPD and cardiovascular diseases, may
A

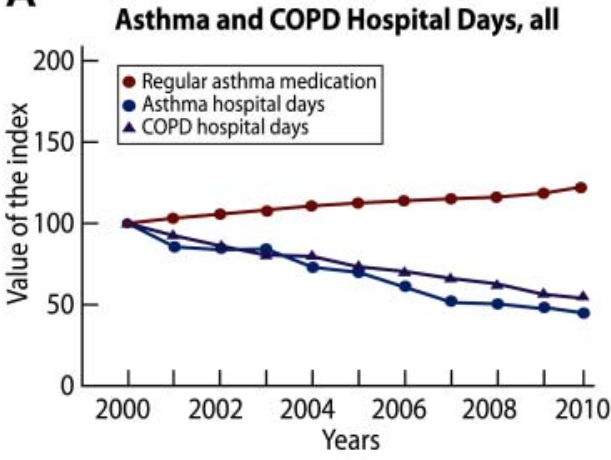

C

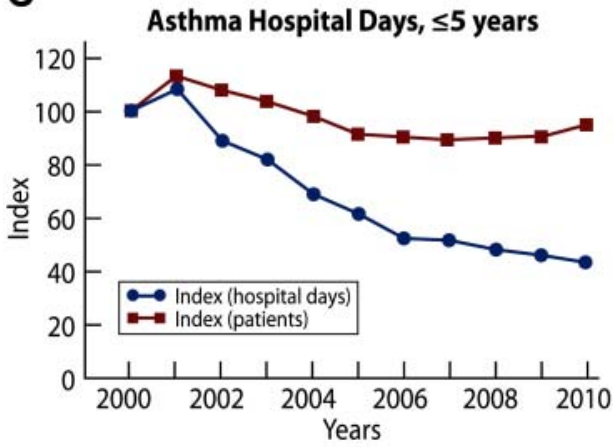

B
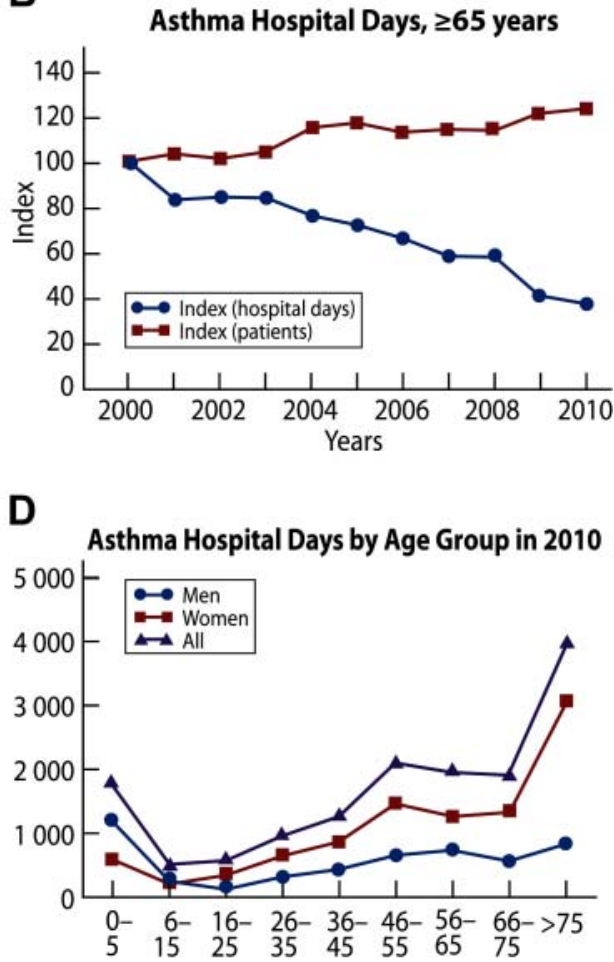

Figure 1 (A) Increase in the number of patients with asthma entitled to special reimbursement for their drug costs and decrease in hospital days due to asthma and chronic obstructive pulmonary disease (COPD). Numbers are relative changes after 2000 (index, 2000=100). Changes in the number hospital days and in the number of asthma patients aged $\geq 65$ years (B) and $\leq 5$ years (C) during the period 2000-2010. (D) Trend for hospital days due to asthma by different age groups in 2010. This figure is only reproduced in colour in the online version. 
complicate treatment in older patients. ${ }^{4} 5$ The other group that needs attention is infants/toddlers who experience wheezing attacks during viral respiratory infections.

The decrease in the hospital days for asthma probably indicates early detection, more effective treatment, and actively implemented guided self-management to prevent exacerbations. In our experience, the latter is the key as it provides simple tools for patients (and parents) to stop asthma attacks proactively. ${ }^{3}$

To conclude, the burden of asthma has continued to decrease in Finland during the last decade as the costs attributed to asthma (including disability, medication, hospital care, outpatient visits) decreased considerably during the period 1994-2004, ${ }^{2}$ and this trend has continued. In older patients, memory impairment and co-morbidities remain as challenges. The Global Initiative for Asthma launched the Asthma Control Challenge in May 2010 with the aim of reducing hospitalisations due to asthma by $50 \%$ over the next 5 years, ${ }^{6}$ and based on the trends observed in our study this seems to be an achievable goal. ${ }^{7}$
Paula Kauppi, ${ }^{1}$ Miika Linna, ${ }^{2}$ Jaana Martikainen, ${ }^{3}$ Mika J Mäkelä, ${ }^{1}$ Tari Haahtela ${ }^{1}$

${ }^{1}$ Department of Allergy, Skin and Allergy Hospital, Helsinki University Central Hospital, Helsinki, Finland ${ }^{2}$ Centre for Health and Social Economics, Institute of Health and Welfare, Helsinki, Finland

${ }^{3}$ Research Department, Social Insurance Institution, Helsinki, Finland

Correspondence to Dr Paula Kauppi, Department of Allergy, Skin and Allergy Hospital, Helsinki University Central Hospital, Meilahdentie 2, PO Box 160, Fl00029 HUS, Helsinki, Finland; paula.kauppi@hus.fi

Contributors PK: analysis of the study, drafting and revising the manuscript. ML and JM: acquisition of data and revising the manuscript. MJM: revising the manuscript. TH: design of the study and revising the manuscript.

Funding This study was supported by the Finnish Allergy Programme of the Ministry of Social Affairs and Health.

\section{Competing interests None.}

Ethics approval This study was approved by the ethical committee of the Helsinki and Uusimaa Hospital District.

Provenance and peer review Not commissioned; externally peer reviewed.

To cite Kauppi P, Linna M, Martikainen J, et al. Thorax 2013;68:292-293.
Accepted 14 March 2012

Published Online First 15 April 2012

Thorax 2013;68:292-293.

doi:10.1136/thoraxjnl-2011-201028

\section{REFERENCES}

1 Pallasaho $P$, Juusela $M$, Lindqvist $A$, et al. Allergic rhinoconjunctivitis doubles the risk for incident asthma -results from a population study in Helsinki, Finland. Respir Med 2011;105:1449-56.

2 Haahtela T, Tuomisto LE, Pietinalho A, et al. A 10 year asthma programme in Finland: a major change for the better. Thorax 2006;61:663-70.

3 Haahtela T, von Hertzen L, Mäkelä M, et al. Allergy programme Working Group. Finnish Allergy programme 2008-2018 - time to act and change the course. Allergy 2008;63:634-45.

4 Kauppi $\mathrm{P}$, Kupiainen $\mathrm{H}$, Lindqvist $\mathrm{A}$, et al. Overlap syndrome of asthma and COPD predicts low quality of life. J Asthma 2011:48:279-85.

5 Gershon AS, Wang C, Guan J, et al. Burden of comorbidity in individuals with asthma. Thorax 2010;65:612-18.

6 FitzGerald JM, Bateman E, Hurd S, et al. The Global Initiative in Asthma (GINA) five year asthma challenge: reducing asthma hospitalizations. Eur Respir 」 2011;38:997-8

7 Kupczyk M, Haahtela T, Cruz AA, et al. Reduction of asthma burden is possible through National Asthma Plans. Allergy 2010;65:415-19. 